Tohoku Math. J.

58 (2005), 89-100

\title{
ON DYNAMICS OF GENERALIZED COMPETITIVE AND COOPERATIVE SYSTEMS
}

\author{
LiHONG HuAng, TAishan Yi AND Xingfu Zou
}

(Received February 10, 2004, revised April 15, 2005)

\begin{abstract}
In this paper, we are concerned with $n$-dimensional generalized competitive or cooperative systems of ordinary differential equations. A result is established to show that the flow generated by a generalized cooperative and irreducible system is strongly monotone. Also, it is shown that an analogue of the Poincarè-Bendixon theorem holds for three dimensional generalized competitive and dissipative systems. Finally, we provide a generalized Smale's construction.
\end{abstract}

1. Introduction. Since the early works of Kamke [6] and Müller [7], monotone dynamical system theory has been showing its power in more and more models described by various differential equations, including ordinary, delay and parabolic differential equations. When considering models of ordinary differential equations, most frequently used ordering in the phase space is the one induced by the first orthant in $\boldsymbol{R}^{n}$. This cone is a natural choice and is especially plausible and convenient in studying population growth models, due to the practical demand on positive invariance of the population density. Systems that are monotone with respect to the ordering induced by this cone have been referred as (classical) cooperativecompetitive systems; see Hirsch's series of works [1-5]. Such systems can demonstrate simple dynamics (e.g., generic convergence) under some extra conditions. In the meantime, they can also allow very complicated behaviour. Indeed, Smale's construction (see Smale [11]) shows that any vector field on the standard $(n-1)$-simplex in $\boldsymbol{R}^{n}$ can be embedded into a smooth competitive vector field on $\boldsymbol{R}^{n}$ for which the simplex is an attractor, which implies that the limiting behaviour of classical competitive systems can be arbitrarily complicated. On the other hand, from Hirsch [1], one knows that a classical competitive or cooperative system in $\boldsymbol{R}^{n}$ behaves essentially like a system in $\boldsymbol{R}^{n-1}$.

As stated in the book by Smith [14], sometimes it is advantageous to consider other orthants (other than $\boldsymbol{R}_{+}^{n}$ ) as order cones. For example, in analyzing a population model of $n$ interacting species where $k$ of which interact with each other in cooperative manner, the remaining $n-k$ species interact with each other in a cooperative manner, but the interaction between any two species in different groups is competitive, Smith $[12,13]$ used the ordering

2000 Mathematics Subject Classification. Primary 34C25; Secondary 34K25, 34C12.

Key words and phrases. Competitive/cooperative system, Smale's construction, dynamics, arbitrary order cone. Research supported by the Natural Science Foundation of China, the Key Project of Chinese Ministry of Education, and the Foundation for University Excellent Teacher by Chinese Ministry of Education (LH); by the Doctorate Program Foundation of Chinese Ministry of Education (TY); and by NSERC of Canada (XZ). 
induced by the corresponding orthant in which the $k$ coordinates are nonnegative and the remaining $n-k$ coordinates are non-positive. This shows that by properly choosing an orthant as the order cone, one may be able to enlarge the class of cooperative and competitive systems. For a detailed discussion on the other orthant cones, the reader is referred to the book by Smith [14, pp. 48-50].

The success of Smith $[12,13,14]$ encourages people to further enlarge the class of cooperative and competitive systems by considering other cones that are not orthants of $\boldsymbol{R}^{n}$. Recently, Ortega and Sánchez [8] examined the monotonicity of solution flows of ordinary differential systems with respect to the ordering induced by the so-called "quadratic cones". In the special case of $n=3$, the resulting monotonicity was then employed to generalize the Poincarè-Bendixon theorem and then to obtain the existence of an orbitally stable closed orbit. The results in Ortega and Sánchez [8] have found an successful application to the Rauch's circuit system in Sánchez [9].

While attempts can be kept making to identifying other cones, one can also simultaneously work in the other direction, that is, studying cooperative and competitive systems with respect to an arbitrary ordering (referred throughout this paper as generalized cooperative and competitive systems). The former requires much knowledge and experience on the models and is much more involved than the standard ordering in $\boldsymbol{R}^{n}$. For the latter, Walcher [16] recently explored such generalized cooperative systems. After clarifying some necessary notions on and establishing the criteria for verification of such generalized cooperative systems, Watcher [16] extended Müller's classical monotonicity theorem and Kamke's comparison theorem to such generalized cooperative systems.

Walcher [16] has only developed conditions for the systems to generate a monotone flow with respect to an arbitrary ordering. However, it is well-known that monotonicity only is not enough to guarantee some nice properties, and in order for the system to have such nice properties, stronger conditions are required among which is the strong monotonicity. For example, while monotonicity does not lead to the "generic convergence", strong monotonicity does; see, e.g., Smith and Thieme [15]. It is known that in the standard ordering case, cooperative property and irreducibility guarantee the strong monotonicity of the flow of the system. One naturally asks what about in a case with an arbitrary ordering? The primary objective of this paper is to introduce the corresponding irreducibility for a system with respect to an arbitrary ordering, by which we will establish the corresponding strong monotonicity for the solution flow.

The second objective of this paper is to discuss Smale's construction, which implies that classical cooperative and irreducible systems in $\boldsymbol{R}^{n}$ can admit any type of dynamic behaviour of systems in $\boldsymbol{R}^{n-1}$, and thus, can demonstrate very complicated dynamics, such as chaos and strange attractors. Smale's classical construction is done under the standard ordering and it heavily depends on the structure of the positive cone $\boldsymbol{R}_{+}^{n}$. Now that one can generalize the classical cooperative and irreducible systems to the ones with respect to arbitrary orderings, and now that one has infinitely many choices for order cones, one may wonder if there is any cone in $\boldsymbol{R}^{n}$ that will induce an ordering with respect to which the corresponding cooperative 
and irreducible systems can only allow certain types (say, simpler ones) of dynamics. In other words, are the implications of Smale's construction ordering dependent? We will give a negative answer to this question by extending Smale's construction to a generalized one.

The rest of this paper is organized as follows. In Section 2, we give some key definitions for generalized competitive, cooperative and irreducible systems, and establish a result (Theorem 2.1) to show that the flow generated by a generalized cooperative and irreducible system is strongly monotone. An example and some discussions are also given to illustrate that our result is different from those in $[3,13]$ and is a generalization of some results in [8]. Section 3 is devoted to demonstrating that the results for the classical competitive-cooperative and irreducible systems given by Smale [11] also hold for generalized competitive or cooperative systems studied in Section 2.

2. Generalized competitive and cooperative systems. In this section, we introduce several concepts and notation which will be used throughout this paper.

Let $K \subset \boldsymbol{R}^{n}$ be a closed cone with nonempty interior and denote by $\operatorname{Int} K$ the interior of $K$ in $\boldsymbol{R}^{n}$. In what follows, $K^{*}$ will be used to denote the dual cone of $K$, i.e., $K^{*}=$ $\left\{\lambda \in \boldsymbol{R}^{n} ;\langle\lambda, x\rangle \geq 0\right.$ for all $\left.x \in K\right\}$, in which $\langle\cdot, \cdot \cdot\rangle$ is the standard inner product in $\boldsymbol{R}^{n}$. For $x, y \in \boldsymbol{R}^{n}$, we denote (i) $x \leq_{K} y$ if and only if $y-x \in K$; (ii) $x<_{K} y$ if and only if $x \leq_{K} y$ and $x \neq y$; and (iii) $x \ll_{K} y$ if and only if $y-x \in \operatorname{Int} K$. We say that $U \subset \boldsymbol{R}^{n}$ is $p$-convex if $t x+(1-t) y \in U$ for all $t \in[0,1]$ whenever $x, y \in U$ and $x \leq_{K} y$.

We need the following key definitions.

DEFINITION 2.1. Let $A$ be an $n \times n$ matrix.

(i) $A$ is said to be cooperative with respect to $K$ if for any $x \in K$ and any $\lambda \in K^{*}$ with $\langle\lambda, x\rangle=0$, we have $\langle\lambda, A x\rangle \geq 0$.

(ii) $A$ is said to be irreducible with respect to $K$ if for any $x \in K \backslash \operatorname{Int} K \backslash\{0\}$, there exists $\lambda \in K^{*}$ such that $\langle\lambda, x\rangle=0$ and $\langle\lambda, A x\rangle \neq 0$ (necessarily, $\lambda \in K^{*} \backslash \operatorname{Int} K \backslash\{0\}$ ).

(iii) $\quad A$ is said to be totally cooperative with respect to $K$ if $A x \in \operatorname{Int} K$ for all $x \in$ $K \backslash\{0\}$.

REMARK 2.1. If $-A$ satisfies the hypotheses of (i) and (iii) respectively, then $A$ is said to be competitive with respect to $K$ and totally competitive with respect to $K$, respectively.

We need the following elementary results whose proofs are contained in $[10,16,17]$.

Lemma 2.1. Let $x \in K$. Then $x \in \operatorname{Int} K$ if and only if $\langle\lambda, x\rangle>0$ for all $\lambda \in K^{*} \backslash\{0\}$.

Lemma 2.2. Let $x \in K \backslash\{0\}$. Then there exists $\lambda \in K^{*}$ such that $\langle\lambda, x\rangle>0$.

Consider an $n$-dimensional autonomous system of ordinary differential equations

$$
\dot{x}(t)=f(x(t)),
$$

where $f: U \longrightarrow \boldsymbol{R}^{n}$ is a continuously differentiable function and $U$ is an open subset of $\boldsymbol{R}^{n}$. We denote by $\varphi_{t}(x)(\varphi)$ or $\left.\varphi(t, x)\right)$ the solution of the initial value problem. It will always 
be assumed that the initial value problem $(2.1)$ with $\varphi_{0}(x)=\varphi(0, x)=x$ which is always assumed to exist globally and uniquely.

Definition 2.2. Let $f$ be defined as above. We say that $f$ is cooperative (irreducible, totally cooperative) with respect to $K$ if for every $x \in U, D f(x)$ is cooperative (irreducible, totally cooperative) with respect to $K$.

THEOREM 2.1. Let $U$ be p-convex and $f$ be cooperative with respect to $K$. Then $\varphi$ is monotone. If, in addition, $f$ is irreducible with respect to $K$, then $\varphi$ is strongly monotone.

PROOF. The first assertion follows from Proposition 1.5 in [16]. We next prove the second assertion. Suppose that $x, y \in U$ with $x>_{K} \quad y$ and set $I_{t}=\left\{\lambda \in K^{*} ;\left\langle\lambda, \varphi_{t}(x)-\right.\right.$ $\left.\left.\varphi_{t}(y)\right\rangle>0\right\}$. Then by Lemma 2.2, we have $I_{t} \neq \phi$ for all $t \in R_{+}$. We will show that $I_{t}=K^{*}$ for all $t>0$. Otherwise, there exists $\delta>0$ such that $K^{*} \backslash I_{\delta} \neq\{0\}$. Since $U$ is $p$-convex, it follows that

$$
f(\varphi(\delta, x))-f(\varphi(\delta, y))=\int_{0}^{1} D f(s \varphi(\delta, x)+(1-s) \varphi(\delta, y))(\varphi(\delta, x)-\varphi(\delta, y)) d s .
$$

Since $f$ is irreducible with respect to $K$, there exists $\lambda \in K^{*} \backslash I_{\delta} \backslash\{0\}$ such that

$$
\langle\lambda,(\varphi(\delta, x)-\varphi(\delta, y))\rangle=0 \quad \text { and }\langle\lambda, D f(\varphi(\delta, x))(\varphi(\delta, x)-\varphi(\delta, y))\rangle>0 .
$$

Again, since $f$ is cooperative with respect to $K$, for the above $\lambda \in K^{*} \backslash I_{\delta} \backslash\{0\}$, we have

$$
\langle\lambda, D f(s \varphi(\delta, x)+(1-s) \varphi(\delta, y))(\varphi(\delta, x)-\varphi(\delta, y))\rangle \geq 0, \quad s \in[0,1] .
$$

It follows that

$$
\left\langle\lambda, \varphi^{\prime}(\delta, x)-\varphi^{\prime}(\delta, y)\right\rangle=\langle\lambda, f(\varphi(\delta, x))-f(\varphi(\delta, y))\rangle>0 .
$$

Hence, from $\langle\lambda, \varphi(\delta, x)-\varphi(\delta, y)\rangle=0$, there exists sufficiently small $\varepsilon>0$ such that

$$
\langle\lambda, \varphi(\delta-\varepsilon, x)-\varphi(\delta-\varepsilon, y)\rangle<0,
$$

from which one can conclude that $(\varphi(\delta-\varepsilon, x)-\varphi(\delta-\varepsilon, y)) \notin K$, a contradiction to the first assertion. From Lemma 2.1, we can deduce that the conclusion of the theorem holds true.

REMARK 2.2. A similar result as Theorem 2.1 holds for a nonautonomous system.

REMARK 2.3. If $-f$ satisfies the hypotheses of Theorem 2.1 , then $f$ is said to be competitive (irreducible, totally competitive) with respect to $K$. Consider the negative flows generated by system (2.1), then it is easy to obtain its related results.

REMARK 2.4. We should mention that many results of Hirsch [1-5] are also true for generalized cooperative or competitive systems. In particular, note that the flow on a compact limit set of a generalized cooperative or competitive system in $\boldsymbol{R}^{n}$ is topologically equivalent to a flow on a compact invariant set of a Lipschitz system of differential equations in $\boldsymbol{R}^{n-1}$, which implies that generalized competitive or cooperative systems can behave no worse than general systems of one less dimension. The arguments in the classical competitive or cooperative systems [1] can be literally exploited to prove this. 
REMARK 2.5. In the case where $f$ is totally cooperative with respect to $K$, it is easy to check that the flow generated by the system (2.1) is strongly monotone. A similar result holds for the case where $f$ is totally competitive with respect to $K$.

To compare our results with those obtained in [3] and [13], we give the following illustrative example.

EXAMPLE 2.1. Consider the following system of 5-dimensional differential equations

$$
\dot{x}(t)=g(x(t)),
$$

where $x(t)=\left(x_{1}(t), x_{2}(t), x_{3}(t), x_{4}(t), x_{5}(t)\right)^{T} \in \boldsymbol{R}^{5}$ and

$$
g\left(x_{1}, x_{2}, \ldots, x_{5}\right)=\left(\begin{array}{l}
x_{1}+x_{2}+\sin a x_{3}+3 x_{5} \\
x_{1}+2 x_{2}+x_{5} \\
x_{5}+\cos b x_{4} \\
0 \\
2 x_{1}+x_{2}+2 x_{5}
\end{array}\right),
$$

where $a \in(-3,3)$ and $b \in(-1,1)$. Set $K_{1}=\left\{\left(x_{1}, x_{2}, \ldots, x_{5}\right)^{T} \in \boldsymbol{R}^{5} ; x_{1} \geq 0, x_{2} \geq 0, x_{5} \geq\right.$ $\left.\sqrt{x_{3}^{2}+x_{4}^{2}}\right\}$, and let $\Phi$ be the flow generated by the system (2.2).

First, we will show that $g$ is cooperative and irreducible with respect to $K_{1}$. Indeed, a direct calculation shows that

$$
D g(x)=\left(\begin{array}{ccccc}
1 & 1 & a \cos a x_{3} & 0 & 3 \\
1 & 2 & 0 & 0 & 1 \\
0 & 0 & 0 & -b \sin b x_{4} & 1 \\
0 & 0 & 0 & 0 & 0 \\
2 & 1 & 0 & 0 & 2
\end{array}\right)
$$

Then for any $y=\left(y_{1}, y_{2}, \ldots, y_{5}\right) \in K_{1} \backslash\{0\}$, we have

$$
D g(x) y=\left(\begin{array}{l}
y_{1}+y_{2}+a y_{3} \cos a x_{3}+3 y_{5} \\
y_{1}+2 y_{2}+y_{5} \\
y_{5}-b y_{4} \sin b x_{4} \\
0 \\
2 y_{1}+y_{2}+2 y_{5}
\end{array}\right) .
$$

It follows that $D g(x) y \in \operatorname{Int} K_{1}$, that is, $g$ is totally cooperative with respect to $K_{1}$. Hence $g$ is cooperative and irreducible with respect to $K_{1}$. Therefore, by Theorem $2.1, \Phi$ is strongly monotone in $\left(\boldsymbol{R}^{5}, K_{1}\right)$. However,

(i) if $a=b=0$, then from the above discussion, we obtain

$$
D g(x)=\left(\begin{array}{lllll}
1 & 1 & 0 & 0 & 3 \\
1 & 2 & 0 & 0 & 1 \\
0 & 0 & 0 & 0 & 1 \\
0 & 0 & 0 & 0 & 0 \\
2 & 1 & 0 & 0 & 2
\end{array}\right)
$$


Hence for any $x \in \boldsymbol{R}^{5}, D g(x)$ is cooperative with respect to $\boldsymbol{R}_{+}^{5}$ but not irreducible, that is, $g$ satisfies the Kamke condition, but $g$ is not irreducible in the sense of Hirsch [3]. Hence we can only conclude that $\Phi$ is monotone (but not necessarily strongly monotone) in the ordered space $\left(\boldsymbol{R}^{5}, \boldsymbol{R}_{+}^{5}\right)$.

(ii) if $a \in(-3,3), b \in(-1,1)$ and $a b \neq 0$, then from the above discussion, $a \cos a x_{3}$ as one of the entries of $D g(x)$ is not sign-stable in $\boldsymbol{R}^{5}$, that is, the Jacobian matrix of $g$ is not sign-stable in $\boldsymbol{R}^{5}$. Hence $g$ does not satisfy the type $K$ condition (see [13, Lemma 2.1]). Therefore, we can not apply the corresponding results of [3] and [13].

In the remaining part of this section, we illustrate that our Theorem 2.1 includes some of the results obtained in Ortega and Sánchez [8] as a special case. To this end, we first recall that (2.1) is said to be dissipative if there exists a compact set $D \subset \boldsymbol{R}^{n}$ such that for every $x \in \boldsymbol{R}^{n}$, $\varphi_{t}(x)=\varphi(t, x)$ is in $D$ for $t$ sufficiently large. Now, combining Theorem 2.1 and Remark 2.3 with Theorem 1 in [8], a generalization of Theorem 3 in [8] can be easily obtained as follows.

COROLLARY 2.1. Let $K_{3}$ be an order cone with nonempty interior. Suppose that $f$ is competitive and irreducible with respect to $K_{3}$, and there exists a unique equilibrium $x=0$ such that the eigenvalues of $D F(0)$ satisfy

$$
\lambda_{1}<0, \quad \operatorname{Re} \lambda_{2}>0, \quad \operatorname{Re} \lambda_{3}>0 .
$$

If (2.1) is dissipative, then there exists at least one orbitally stable closed orbit. Moreover, every orbit tends to the equilibrium or to a closed orbit as $t \longrightarrow+\infty$.

REMARK 2.6. For some applications of Corollary 2.1, we refer the reader to [8,9].

Let $S$ be an $n \times n$ symmetric matrix having one positive eigenvalue and $n-1$ negative eigenvalues. Let $\lambda_{+}$denote the positive eigenvalue and $e_{+}$be an eigenvector satisfying

$$
S e_{+}=\lambda_{+} e_{+}, \quad\left\|e_{+}\right\|=1 .
$$

Define the set

$$
K_{S}=\left\{\xi \in \boldsymbol{R}^{n} ;\langle S \xi, \xi\rangle \geq 0,\left\langle\xi, e_{+}\right\rangle \geq 0\right\} .
$$

Set $K=K_{S}$, which we call a quadratic cone. As stated in [8], $K$ is an order cone with nonempty interior. Hence, $K$ induces a strongly ordered space $\left(\boldsymbol{R}^{n}, K\right)$. Let $A$ be an $n \times n$ matrix. Then we shall say that $A$ is (strictly) $S$-competitive if there exists $\lambda \in R$ such that $S A+A^{T} S+\lambda S$ is negative (definite) semidefinite, where $A^{T}$ denotes the transposed matrix of $A$. We shall say that $f$ is (strictly) $S$-competitive if $D f(\xi)(\xi \in U)$ is (strictly) $S$-competitive. The flow $\Phi$ generated by system (2.1) is said to be strongly $L$-monotone (in the past) if for each $\xi \in \boldsymbol{R}^{n}$ and $\delta \in K \backslash\{0\}$ one has $D \Phi_{t}(\xi) \delta \gg 0$ for all $t<0$.

We now establish two crucial lemmas.

LEMma 2.3. Let $K_{S}^{*}$ be the dual cone of $K_{S}$. Then $K_{S}^{*}=S K_{S}$.

Proof. Since $S$ is symmetric, there exists an orthogonal matrix $T$ such that $T^{-1} S T=$ $\operatorname{diag}\left(\lambda_{1}, \lambda_{2}, \ldots, \lambda_{n}\right) \equiv S_{1}$, where $\lambda_{1}=\lambda_{+}>0$ and $\lambda_{i}<0, i=2, \ldots, n$. By the definition 
of $K_{S}$, we obtain

$$
K_{S}=\left\{\xi \in \boldsymbol{R}^{n} ;\left\langle S_{1} T^{-1} \xi, T^{-1} \xi\right\rangle \geq 0,\left\langle T^{-1} \xi, T^{-1} e_{+}\right\rangle \geq 0\right\} .
$$

Hence $K_{S}=T K_{S_{1}}$. From $K_{S_{1}}^{*}=S_{1} K_{S_{1}}$, where $K_{S_{1}}^{*}$ denotes the dual cone of $K_{S_{1}}$, it follows that $K_{S}^{*}=T K_{S_{1}}^{*}=T S_{1} K_{S_{1}}$, that is, $K_{S}^{*}=T S_{1} T^{-1} K_{S}=S K_{S}$. The proof is complete.

LEMmA 2.4. Let A be an $n \times n$ matrix. Then

(i) $A$ is competitive with respect to $K$ if and only if $A$ is $S$-competitive;

(ii) $A$ is competitive and irreducible with respect to $K$ if and only if $A$ is strictly $S$ competitive.

ProOF. We only prove (ii). The proof of (i) is similar, and thus is omitted. Sufficiency is straightforward. To prove necessity, let us assume that $A$ is competitive and irreducible with respect to $K$. Let $K^{*}$ be the dual cone of $K$. Then by Lemma 2.3, we obtain $K^{*}=$ $S K=\{S \xi ; \xi \in K\}$. Define the set $K_{\xi}=\left\{\eta \in K^{*} ;\langle\eta, \xi\rangle=0\right\}$. Then

$$
K_{\xi}= \begin{cases}\phi & \text { for } \xi \in \operatorname{Int} K, \\ \{S \xi\} & \text { for } \xi \in \partial K \backslash\{0\}, \\ K^{*} & \text { for } \xi=0,\end{cases}
$$

where $\partial K$ denotes the boundary of $K$. Since $A$ is competitive and irreducible with respect to $K$, for any $\xi \in K \backslash\{0\}$ with $\langle S \xi, \xi\rangle=0$ (which implies $\xi \in K \cup(-K)$, we have $\langle S \xi, A \xi\rangle<0$. Hence, for any $\xi \in \boldsymbol{R}^{n} \backslash\{0\}$ with $\langle S \xi, \xi\rangle=0$, we can obtain that $\left\langle\left(S A+A^{T} S\right) \xi\right.$, $\left.\xi\right\rangle<0$. It follows from Lemma 1 and its related illustrations in [8] that there exists $\lambda \in R$ such that $S A+A^{T} S+\lambda S$ is negative definite, namely, $A$ is strictly $S$-competitive. This completes the proof of the lemma.

An application of Lemma 2.4, together with Theorem 2.1 and Remark 2.3, yields an immediate consequence below, which is in fact one of the main results of [8], that is, Theorem 2 in [8].

COROLlary 2.2. Let $f$ and $S$ be defined as above. Then $f$ is $S$-competitive if and only if the flow generated by (2.1) is monotone in the past (with respect to $K$ ). Moreover, if $f$ is strictly S-competitive, then the flow is strongly L-monotone.

3. Generalized Smale's construction. As noted in Section 1, Smale [11] has pointed out that a smooth system can be embedded in a classical competitive and smooth system. On the other hand, as mentioned in Remark 2.4, generalized competitive or cooperative systems in $\boldsymbol{R}^{n}$ can not behave worse than general systems in $\boldsymbol{R}^{n-1}$. In consequence, any thoughts that we can provide a complete description of the dynamics generated by the classical competitive and smooth systems have to be scrapped. It is natural to ask whether similar conditions are to be found for generalized competitive and cooperative systems. To be more precise, when an order cone $K$ is given, is there any hope for cooresponding competitive or cooperative systems to have simple dynamics? In this section we provide a negative answer to this question. Indeed, 
we present a generalized Smale's construction and hence show that generalized competitive or cooperative systems in $\boldsymbol{R}^{n}$ can also not behave simpler than general systems in $\boldsymbol{R}^{n-1}$.

Before continuing, some definitions and preliminary results are necessary.

Definition 3.1. Let $K \subset \boldsymbol{R}^{n}$ be an order cone with nonempty interior and suppose that $\lambda \in K^{*}$. We say that $\lambda$ is an admitting element of $K$ if $\lambda \in \operatorname{Int} K \cap \operatorname{Int} K^{*}$.

REMARK 3.1. It is obvious from the separation theorem that an admitting element of $K$ must exist. See the proof of Lemma 3.2 below.

Definition 3.2. Let $K \subset \boldsymbol{R}^{n}$ be an order cone with nonempty interior. $K$ is called an admitting cone if $\lambda=(0,0, \ldots, 0,1)^{T} \in \boldsymbol{R}^{n}$ is an admitting element of $K$.

Definition 3.3. Let $K_{1}, K_{2} \subset \boldsymbol{R}^{n}$ be order cones with nonempty interior. Then $K_{1}$ and $K_{2}$ are said to be isomorphic if there exists an orthogonal transfomation $T: \boldsymbol{R}^{n} \rightarrow \boldsymbol{R}^{n}$ such that $T K_{1}=K_{2}$. We say that such $T$ is an isomorphic map from the cones $K_{1}$ to $K_{2}$.

Lemma 3.1. Let $A$ be an $n \times n$ matrix. Suppose that $K_{1} \subset \boldsymbol{R}^{n}$ and $K_{2} \subset \boldsymbol{R}^{n}$ are isomorphic order cones and define $T$ as in Definition 3.3. If A are cooperative (irreducible, totally cooperative) with respect to $K_{1}$, then $T A T^{-1}$ are also cooperative (irreducible, totally cooperative) with respect to $K_{2}$.

Proof. We only consider the case when $A$ is cooperative with respect to $K_{1}$, the other cases being similar. Let $K_{1}^{*}$ and $K_{2}^{*}$ denote the dual cones of $K_{1}$ and $K_{2}$, respectively. Since $K_{2}=T K_{1}$ and $T$ is orthogonal, it follows that $K_{2}^{*}=T K_{1}^{*}$. Suppose that $A$ is cooperative with respect to $K_{1}$, and $\lambda_{2} \in K_{2}^{*}$ and $x_{2} \in K_{2}$ with $\left\langle\lambda_{2}, x_{2}\right\rangle=0$. Then there exist $\lambda_{1} \in K_{1}^{*}$ and $x_{1} \in K_{1}$ such that

$$
x_{2}=T x_{1}, \quad \lambda_{2}=T \lambda_{1} .
$$

Thus,

$$
\left\langle\lambda_{1}, x_{1}\right\rangle=\left\langle\lambda_{2}, x_{2}\right\rangle=0 .
$$

It follows that $\left\langle\lambda_{1}, A x_{1}\right\rangle \geq 0$, and hence, $\left\langle T \lambda_{1}, T A T^{-1} T x_{1}\right\rangle \geq 0$. Therefore, $\left\langle T \lambda_{1}, T A T^{-1} x_{2}\right\rangle$ $\geq 0$, which implies that $T A T^{-1}$ is cooperative with respect to $K_{2}$. The proof of the lemma is complete.

REMARK 3.2. It should be noted that "cooperative" in Lemma 3.1 can be replaced by "competitive".

LemmA 3.2. Let $K_{1} \subset \boldsymbol{R}^{n}$ be an order cone with nonempty interior. Then there exists an admitting cone $K_{2}$ in $\boldsymbol{R}^{n}$ such that $K_{1}$ and $K_{2}$ are isomorphic.

Proof. We assume that $K_{1}^{*}$ is the dual cone of $K_{1}$, and hence $\operatorname{Int} K_{1}^{*} \neq \phi$. We will show that $\operatorname{Int} K_{1} \cap \operatorname{Int} K_{1}^{*} \neq \phi$. Otherwise, by the convex separation theorem, there exists $\mu \in \boldsymbol{R}^{n} \backslash\{0\}$ such that $\left\langle\mu, \operatorname{Int} K_{1}\right\rangle \geq 0$ and $\left\langle\mu, \operatorname{Int} K_{1}^{*}\right\rangle \leq 0$. Thus, $\mu \in K_{1}^{*}$ and $\left\langle\mu, K_{1}^{*}\right\rangle \leq 0$. It follows that $\langle\mu, \mu\rangle \leq 0$, that is, $\mu=0$, a contradiction. So we can choose $\lambda \in \operatorname{Int} K_{1} \cap \operatorname{Int} K_{1}^{*}$, which implies that $\lambda$ is an admitting element of $K_{1}$. Let an orthogonal transformation $T$ : 
$\boldsymbol{R}^{n} \rightarrow \boldsymbol{R}^{n}$ be defined by

$$
T \lambda=(0,0, \ldots, 0,1)^{T} \in \boldsymbol{R}^{n}
$$

and

$$
T\left(\left\{x \in \boldsymbol{R}^{n} ;\langle\lambda, x\rangle=0\right\}\right)=\left\{x \in \boldsymbol{R}^{n} ; x_{n}=0\right\} .
$$

Let $K_{2}=T K_{1}$. Then $K_{2}$ is an admitting cone in $\boldsymbol{R}^{n}$, and $K_{1}$ and $K_{2}$ are isomorphic. This completes the proof.

LEMmA 3.3. Let $K$ be an admitting cone in $\boldsymbol{R}^{n}$. Then the set $\left\{x \in K ; x_{n}=1\right\}$ is a closed and bounded set of $\boldsymbol{R}^{n}$.

Proof. Set $G=\left\{x \in K ; x_{n}=1\right\}$. Clearly, $G$ is a closed set. We next show that $G$ is bounded. Otherwise, there exists $x^{(i)} \in G$ such that $\left\|x^{(i)}\right\| \rightarrow+\infty$. Let $y^{(i)}=x^{(i)} /\left\|x^{(i)}\right\| \in$ $K$. Then we have $y^{(i)} \rightarrow y$ for some $y=\left(y_{1}, \ldots, y_{n}\right)$ as $i \rightarrow \infty$ (if necessary, we may choose a subsequence of $\left.y^{(i)}\right)$. Thus, $y \in K$ and $y_{n}=0$. Since $\lambda=(0,0, \ldots, 0,1)^{T} \in \boldsymbol{R}^{n}$ is an admitting element of $K$, it follows that $0=\langle y, \lambda\rangle>0$, a contradiction. This completes the proof.

LEMMA 3.4. Let $f: V \rightarrow \boldsymbol{R}^{n}$ be continuously differentiable, where $V$ is a subset in $\boldsymbol{R}^{n}$, and let $M=\sup _{x \in V, 1 \leq i, j \leq n}\left|(D f(x))_{i j}\right|$. Suppose that $M<+\infty$ and $K$ is an admitting cone in $\boldsymbol{R}^{n+1}$. Then there exists a continuously differentiable function $g: V \times R^{1} \rightarrow \boldsymbol{R}^{n+1}$ such that $g$ is totally cooperative (totally competitive) with respect to $K$, and $\left.g\right|_{V \times\{0\}}=f$.

PRoOF. Let $g_{\alpha}: V \times R^{1} \rightarrow \boldsymbol{R}^{n+1}$ be defined by

$$
g_{\alpha}=\left(x_{1}, x_{2}, \ldots, x_{n}, x_{n+1}\right)=\left(f\left(x_{1}, x_{2}, \ldots, x_{n}\right), \alpha x_{n+1}\right) .
$$

Then for any $\alpha \in R$, one sees that $g_{\alpha}$ is a smooth function and $\left.g_{\alpha}\right|_{V \times\{0\}}=f$. Since

$$
D g_{\alpha}(x)=\left(\begin{array}{cc}
D f\left(x_{1}, x_{2}, \ldots, x_{n}\right) & 0 \\
0 & \alpha
\end{array}\right)
$$

and $K$ is an admitting cone in $\boldsymbol{R}^{n+1}$, it follows that $\lambda=(0,0, \ldots, 0,1) \in \boldsymbol{R}^{n+1}$ is an admitting element of $K$. By $M<+\infty$ and Lemma 3.3, one can obtain that there exists $\alpha_{1}>0$ such that for any $x \in V \times R, D g_{\alpha_{1}}(x)$ is totally cooperative with respect to $K$. Similarly, there exists $\alpha_{2}<0$ such that for any $x \in V \times R, D g_{\alpha_{2}}(x)$ is totally competitive with respect to $K$. Therefore, $g_{\alpha_{1}}$ is totally cooperative with respect to $K$ and $g_{\alpha_{2}}$ is totally competitive with respect to $K$. This completes the proof.

In what follows, we will always assume that $K$ is an order cone with nonempty interior in $\boldsymbol{R}^{n+1}$ and $\lambda \in \boldsymbol{R}^{n+1}$. Let $\langle\lambda, \lambda\rangle=1$ and let $\lambda$ be an admitting element of $K$. Set $L=\left\{x \in \boldsymbol{R}^{n+1} ;\langle\lambda, x\rangle=0\right\}$. In addition, assume that $f: U \rightarrow L$ is a continuously differentiable function, where $U$ is a subset of the hyperplane $L$. We now make the key definition: $H_{U}=\left\{x \in \boldsymbol{R}^{n+1} ;(x-\langle\lambda, x\rangle \cdot \lambda) \in U\right\}=\{x+a \lambda ; x \in U, a \in \boldsymbol{R}\}$.

THEOREM 3.1. Let $K$ and $f$ be defined as above. Suppose that $M=\sup _{x \in U, 1 \leq i, j \leq n}$ $\left|(D f(x))_{i j}\right|<+\infty$. Then there exists a continuously differentiable function $g: H_{U} \rightarrow \boldsymbol{R}^{n+1}$ such that $\left.g\right|_{U}=f$, and $g$ is totally cooperative (totally competitive) with respect to $K$. 
Proof. By Lemma 3.2 and its argument, there exists an admitting cone $K_{1}$ and an orthogonal transformation $T: \boldsymbol{R}^{n+1} \rightarrow \boldsymbol{R}^{n+1}$ such that $T(L)=\left\{x \in \boldsymbol{R}^{n+1} ; x_{n+1}=0\right\}$, $T(\lambda)=(0,0, \ldots, 0,1)^{T}$, and $T K=K_{1}$. It follows that $T U \subset\left\{x \in \boldsymbol{R}^{n+1} ; x_{n+1}=0\right\}$. Define $\prod: \boldsymbol{R}^{n+1} \rightarrow \boldsymbol{R}^{n}$ and $\amalg: \boldsymbol{R}^{n} \rightarrow \boldsymbol{R}^{n+1}$ by $\prod\left(x_{1}, x_{2}, \ldots, x_{n+1}\right)^{T}=\left(x_{1}, x_{2}, \ldots, x_{n}\right)^{T}$ and $\amalg\left(x_{1}, x_{2}, \ldots, x_{n}\right)^{T}=\left(x_{1}, x_{2}, \ldots, x_{n}, 0\right)^{T}$, respectively. Let $V=\prod(T U)$ and let $f_{1}$ : $V \rightarrow \boldsymbol{R}^{n}$ be defined by $f_{1}(x)=\prod \circ T \circ f \circ T^{-1} \circ \bigsqcup(x)$. Then, applying Lemma 3.4 to $f_{1}$ and $K_{1}$, we obtain that there exists $g_{1}: V \times R^{1} \rightarrow \boldsymbol{R}^{n+1}$ such that $g_{1}$ is defined as Lemma 3.4. Define $g: H_{U} \rightarrow \boldsymbol{R}^{n+1}$ by $g(x)=T \circ g_{1} \circ T^{-1}(x)$. Then by Lemma 3.1, $g$ satisfies the conditions of Theorem 3.1, and hence the conclusion of Theorem 3.1 holds true.

REMARK 3.3. It is worth noting that in Theorem 3.1, if $U$ is compact in $\boldsymbol{R}^{n+1}$, then the condition $M<+\infty$ automatically holds.

THEOREM 3.2. Let $f$ and $g$ be as in Theorem 3.1. Suppose that the set $U$ is positively invariant for the system

$$
\dot{x}(t)=f(x(t)) .
$$

Then the set $H_{U}=\left\{x \in \boldsymbol{R}^{n+1} ;(x-\langle\lambda, x\rangle \cdot \lambda) \in U\right\}$ is invariant for the system

$$
\dot{x}(t)=g(x(t)) .
$$

If, in addition, $g$ is totally cooperative (totally competitive) with respect to $K$, then $U$ is a repeller (attractor) for the flows generated by the systems (3.2), and the system (3.2) defined in $U$ is equivalent to the system (3.1).

PROOF. By the preceding arguments in the proof of Theorem 3.1, we only need to verify the theorem under the condition that $f$ and $g$ are defined as in Lemma 3.4. In this case, we also assume that $U \subset \boldsymbol{R}^{n}$ is invariant for the system (3.1). From the choice of $g$ (see Lemma 3.4), it follows that $H_{U}=\left\{x \in \boldsymbol{R}^{n+1} ;\left(x_{1}, x_{2}, \ldots, x_{n+1}\right)\right\}$ is invariant for the system (3.2). If $g$ is totally cooperative with respect to $K$, then from the choice of $g$ (see Lemma 3.4), we can obtain $\alpha>0$, and hence $U \times\{0\}$ is a repeller for the flows generated by the system (3.2). If $g$ is totally competitive with respect to $K$, then we can similarly obtain that $U \times\{0\}$ is an attractor for the flows generated by the system (3.2). Finally, again from the choice of $g$ (see Lemma 3.4), the system (3.2) defined in $U \times\{0\}$ is equivalent to the system (3.1). The proof of the theorem is complete.

REMARK 3.4. It is obvious from Theorem 3.2 that the dynamical properties of an $n$-dimensional dynamical system can be possessed by an $(n+1)$-dimensional generalized competitive or cooperative system. In other words, an $(n+1)$-dimensional generalized competitive or cooperative system at least shares common dynamical properties of a system of differential equations in one less dimension. Again, according to Remark 2.4 and the discussion in Section 2, the dynamics of an $n$-dimensional generalized competitive or cooperative system is essentially equivalent to the dynamics of a general system of one less dimension. Therefore, we can rule out the possibility of generalized competitive or cooperative systems 
having simple dynamics without any additional hypotheses, which implies that the results of Smale [11] also hold for generalized competitive or cooperative systems.

THEOREM 3.3. Let $K$ and $\lambda$ be as in Theorem 3.1. Suppose, for $r>0$, that $L_{r}=$ $\left\{x \in \boldsymbol{R}^{n+1} ;\langle\lambda, x\rangle=r\right\}$. Let $f: L_{r} \cap K \rightarrow L$ be continuously differentiable. Then there exists a continuously differentiable function $g: K \rightarrow \boldsymbol{R}^{n}$ such that $\left.g\right|_{L_{r} \cap K}=f$, and $g$ is totally cooperative (totally competitive) with respect to $K$.

PROOF. We may assume that $K$ is an admitting cone in $\boldsymbol{R}^{n+1}$. Otherwise, using a similar argument to that for the proof of Theorem 3.1, we can show the conclusion is true. In this case where $K$ is an admitting cone, $L_{r}=\left\{x \in \boldsymbol{R}^{n+1} ; x_{n+1}=r\right\}$. We can choose $\eta$ : $\boldsymbol{R} \rightarrow[0,1]$ such that $\eta$ is continuously differentiable, $\eta((-\infty, r / 3]) \equiv 0, \eta([5 r / 3, \infty)) \equiv 0$ and $\eta([2 r / 3,4 r / 3]) \equiv 1$. Define $g_{\beta}: K \rightarrow \boldsymbol{R}^{n+1}$ by

$$
g_{\beta}\left(\left(x_{1}, \ldots, x_{n}, x_{n+1}\right)\right)=\left(\eta\left(x_{n+1}\right) f\left(\frac{r x_{1}}{x_{n+1}}, \ldots, \frac{r x_{n}}{x_{n+1}}\right), \beta\left(x_{n+1}-r\right)\right)^{T} .
$$

Set

and let

$$
\tilde{f}\left(x_{1}, \ldots, x_{n}, x_{n+1}\right)=\eta\left(x_{n+1}\right) f\left(\frac{r x_{1}}{x_{n+1}}, \ldots, \frac{r x_{n}}{x_{n+1}}\right),
$$

$$
M=\sup _{x \in L_{r} \cap K, 1 \leq i, j \leq n}\left|(D \tilde{f}(x))_{i j}\right| .
$$

Clearly, $M<+\infty$. A similar argument as in Lemma 3.4 implies that there exists $\beta>0$ such that $g_{\beta}$ is totally cooperative with respect to $K$, and $\left.g_{\beta}\right|_{L_{r} \cap K}=f$. Similarly, there exists $\beta<0$ such that $g_{\beta}$ is totally competitive with respect to $K$, and $\left.g_{\beta}\right|_{L_{r} \cap K}=f$. This completes the proof.

The following corollary is in fact the main result of Smale [11].

Corollary 3.1. Let $K=\boldsymbol{R}_{+}^{n+1}, \lambda=(1 / \sqrt{n}, \ldots, 1 / \sqrt{n})^{T} \in \boldsymbol{R}_{+}^{n+1}$, and $r=$ $1 / \sqrt{n}$. Suppose that $f$ in Theorem 3.3 can be represented by $f=\left(f_{1}, \ldots, f_{n+1}\right)$ and $f_{i}\left(x_{1}, \ldots, x_{n+1}\right)=x_{i} \widetilde{f}_{i}\left(x_{1}, \ldots, x_{n+1}\right)$, where $\tilde{f}_{i}$ is continuously differentiable. Then there exists $M_{i}: \boldsymbol{R}_{+}^{n+1} \rightarrow \boldsymbol{R}^{n+1}$ such that $\left.M_{i}\right|_{L_{r} \cap K}=\tilde{f}_{i}$ and either $\partial M_{i} / \partial x_{j}>0$ for all $i, j \in\{1,2, \ldots, n\}$ and $x \in K$, or $\partial M_{i} / \partial x_{j}<0$ for all $i, j \in\{1,2, \ldots, n\}$ and $x \in K$. Moreover, the following system

$$
\dot{x}_{i}(t)=x_{i}(t) M_{i}(x(t)), \quad i=1,2, \ldots, n+1,
$$

is totally cooperative (totally competitive) with respect to $K$, and $\triangle^{n}$ is a repeller (attractor) for the system, where $\Delta^{n}=\left\{x \in \boldsymbol{R}_{+}^{n+1} ; \sum x_{i}=1\right\}$.

PROOF. This corollary is a simple consequence of Theorem 3.3.

Acknowledgment. The first author would like to thank the Department of Mathematics and Statistics at Memorial University of Newfoundland for the excellent facilities and support during his visit when this work was carried out. 


\section{REFERENCES}

[ 1 ] M. W. HIRSCH, Systems of differential equations which are cooperative or competitive I, Limit sets, SIAM J. Math. Anal. 13 (1982), 167-179.

[2 ] M. W. HiRsch, The dynamical systems approach to differential equations, Bull. Amer. Math. Soc. (N. S.) 11 (1984), 1-64.

[ 3 ] M. W. HIRSCH, Systems of differential equations that are competitive or cooperative II, Convergence almost everywhere, SIAM J. Math. Anal. 16 (1985), 423-439.

[ 4 ] M. W. HIRSCH, Systems of differential equations that are competitive or cooperative IV, Structural stability in three-dimensional systems, SIAM J. Math. Anal. 21 (1990), 1225-1234.

[ 5 ] M. W. HiRSCH, Systems of differential equations that are competitive or cooperative V, Convergence in 3dimensional systems, J. Differential Equations 80 (1989), 94-106.

[ 6 ] E. KamKe, Zur theorie der systeme gewoknlicher differentialgleichungen, II, Acta Math. 58 (1932), 57-85.

[ 7 ] M. MÜLLER, Über das Fundamenthaltheorem in der Theorie der gewöhnlichen Differentialgleichungen, Math. Z. 26 (1927), 619-645.

[ 8 ] R. Ortega and L. Á. SÁnchez, Abstract competitive systems and orbital stability in $R^{3}$, Proc. Amer. Math. Soc. 128 (2000), 2911-2919.

[9] L. Á. SÁnchez, An application of the theory of monotone systems to an electrical circuit, Proc. Roy. Soc. Edinburgh Sect. A 132 (2002), 711-728.

[10] H. Schaefer, Topological vector spaces, The Macmillan Co., New York, Collier-Macmillan Ltd., London, 1966.

[11] S. Smale, On the differential equations of species in competition, J. Math. Biol. 3 (1976), 5-7.

[12] H. L. Smith, Competing subcommunities of mutualists and a generalized Kamke theorem, SIAM J. Appl. Math. 46 (1986), 856-874.

[13] H. L. SмIтH, System of ordinary differential equations which generate an order preserving flow, A survey of results, SIAM Rev. 30 (1988), 87-113.

[14] H. L. Sмith, Monotone dynamical systems, An introduction to the theory of competitive and cooperative systems, Math. Surveys Monogr. 41, Amer. Math. Soc., Providence, R. I., 1995.

[15] H. L. Smith And H. Thieme, Convergence for strongly order-preserving semiflows, SIAM J. Math. Anal. 22 (1991), 1081-1101.

[16] S. WALChER, On cooperative systems with respect to arbitrary orderings, J. Math. Anal. Appl. 263 (2001), 543-554.

COLLEge OF MATHEMATICS AND ECONOMETRICS

HUNAN UNIVERSITY

Changsha, Hunan 410082

P. R. CHINA

E-mail address: 1hhuang@hnu.net.cn

COLLEGE OF MATHEMATICS AND ECONOMETRICS

HUNAN UNIVERSITY

Changsha, Hunan 410082

P. R. CHINA

E-mail address: yitaishan@163.net

Department of Applied Mathematics

UNIVERSITY OF WESTERN ONTARIO

LONDON, ONTARIO, N6A 5B7

CANADA

E-mail address:xzou@uwo.ca 